Methods: We have retrospectively analyzed the 10-year risk of major osteoporotic fracture calculated with the DeFRAcalc79 tool in postmenopausal women aged over 50 years that were initiating an anti-osteoporotic treatment (fully reimbursed according to the Nota 79). DeFRAcalc79 is a new web-based fracture risk-assessment tool (https://defra-osteoporosi.it) that arithmetically adjusts the risk based on multiple risk factors contemplated by the Nota 79 , which regulates the reimbursability for osteoporosis medications in Italy (Italian Agency for Drugs, AIFA), including demographic and anthropometric data, femoral and/or lumbar spine BMD T-score, family history of femoral or vertebral fractures, number and site of previous osteoporotic fracture (including vertebral, femoral, and non-vertebral non-femoral fractures), glucocorticoid treatment (> 3 or $>12$ months, $\geq 5 \mathrm{mg}$ prednisone or equivalent), adjuvant hormone therapy for breast cancer, and comorbidities that induce an increased risk of fracture (rheumatoid arthritis and other connective tissue diseases, chronic obstructive pulmonary disease, inflammatory bowel diseases, Parkinson's disease, multiple sclerosis, human immunodeficiency virus infection, diabetes, or severe physical handicap). This is a sub-analysis of the cross-sectional observational study to validate and further develop the DeFRA algorithm for the estimation of the risk of osteoporotic fractures, promoted by Verona hospital with the unconditional support of Amgen Srl.

Results: Among 208 women, 116 (55.8\%) were treated with adjuvant hormone therapy for breast cancer and $92(44.2 \%)$ were on glucocorticoid $\geq 5 \mathrm{mg} /$ day. Women on glucocorticoids had a greater mean 10-year risk of fracture compared to women on adjuvant hormone therapy for breast cancer $(67.0 \%$ vs $39.1 \%$ $\mathrm{p}<0.01$ ). $50.7 \%$ of women on adjuvant hormone therapy for breast cancer used denosumab, $28.0 \%$ zoledronic acid and $17.3 \%$ alendronate. In glucocorticoid-induced osteoporosis, $17.6 \%$ of the women used teriparatide, $37.3 \%$ alendronate, $29.4 \%$ zoledronic acid and $13.7 \%$ denosumab.

Conclusion: In our cohort of patients, treatment with adjuvant hormone therapy for breast cancer was slightly more common than glucocorticoids. Women with glucocorticoid-induced osteoporosis had a greater risk of fracture compared to patients treated with adjuvant hormone therapy for breast cancer. Half of the patients on adjuvant hormone therapy for breast cancer were prescribed with denosumab. One-fifth of the patients with glucocorticoid-induced osteoporosis was treated with teriparatide. DeFRAcalc79 is a useful and practical tool for the integrated evaluation of fracture risk in drug-induced osteoporosis.

Disclosure of Interests: Giovanni Adami: None declared, Angelo Fassio Speakers bureau: Angelo Fassio reports personal fees from: Abiogen and Novartis, outside the submitted work., Alessandro Giollo: None declared, Giovanni Orsolini: None declared, Ombretta Viapiana: None declared, Davide Gatti Speakers bureau: Davide Gatti reports personal fees from Abiogen, Amgen, Janssen-Cilag, Mundipharma, outside the submitted work., Maurizio Rossini Speakers bureau: AbbVie, Abiogen, Amgen, BMS, Eli-Lilly, Novartis, Pfizer, Sanofi, Sandoz and UCB DOI: 10.1136/annrheumdis-2020-eular.2565

\section{SAT0457 PREVALENCE OF OSTEOPOROSIS IN OSTEOARTHRITIC PATIENTS: A SYSTEMATIC REVIEW}

A. Ahmadi Pirshahid ${ }^{1}$, D. Kim ${ }^{1}$, Y. Li ${ }^{1}$, T. Varghese ${ }^{1}$, J. Pope ${ }^{1} .{ }^{1}$ Western University, Medicine, London, Canada

Background: There is controversy regarding the relationship between osteoarthritis (OA) and osteoporosis (OP). While OA may be associated with increased bone mineral density $(B M D)$ due to increased weight, evidence exists that the incidence of OP may be increased in patients with OA.

Objectives: To determine whether the prevalence of OP is increased in patients with $\mathrm{OA}$, compared to age and sex-matched populations.

Methods: We conducted a systematic literature review using the databases PubMed, Embase, Scopus, and Web of Science, including articles that that analysed the frequency, rate, prevalence, incidence, risk, or excess risk of OP in patients with OA compared to age and sex-matched comparison groups (controls). Articles with fewer than 200 participants, and those without controls were excluded. Two reviewers conducted title and abstract screening.

Results: Of 2772 unique articles, 49 articles were chosen for full article screening, and 4 articles met the inclusion criteria of our present study. Data from 2 and 4 studies used OP in men and women, respectively. Other articles reported on BMD and not OP so they were excluded. In women, 998 participants with OA were compared with 1903 controls. The pooled estimate of the odds ratio for prevalence of OP vs general matched population was not statistically different (Figure 1). In men, 136 participants with OA were compared with 682 controls. The results did not show a statistically significant different in the frequency of $O P$ in $\mathrm{OA}$ in men (Figure 2).

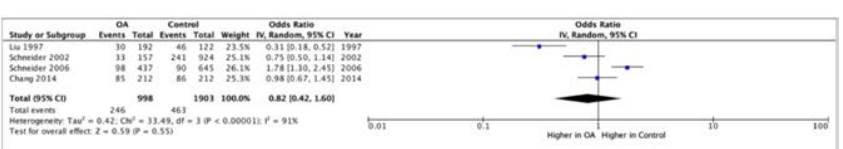

Figure 1. Prevalence of OP in women with OA compared to controls

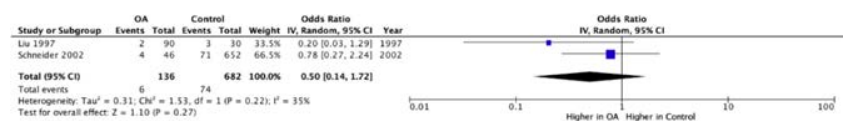

Figure 2. Prevalence of OP in men with $\mathrm{OA}$ compared to controls

Conclusion: The frequency of OP in participants with OA was the same in both men and women compared to the matched controls.

References:

[1] Chang, C. B., Kim, T. K., Kang, Y. G., Seong, S. C., \& Kang, S. B. (2014). Prevalence of osteoporosis in female patients with advanced knee osteoarthritis undergoing total knee arthroplasty. Journal of Korean Medical Science, 29(10), 1425-1431.

[2] Liu, G., Peacock, M., Eilam, O., Dorulla, G., Braunstein, E., \& Johnston, C. C. (1997). Effect of osteoarthritis in the lumbar spine and hip on bone minera density and diagnosis of osteoporosis in elderly men and women. Osteoporosis International, 7(6), 564-569.

[3] Schneider, D. L., Barrett-Connor, E., Morton, D. J., \& Weisman, M. (2002) Bone mineral density and clinical hand osteoarthritis in elderly men and women: the Rancho Bernardo study. The Journal of Rheumatology, 29(7), 1467-1472.

[4] Schneider, D. L., Bettencourt, R., \& Barrett-Connor, E. (2006). Clinical utility of spine bone density in elderly women. Journal of Clinical Densitometry, 9(3), 255-260.

Disclosure of Interests: Ali Ahmadi Pirshahid: None declared, Dongkeun Kim: None declared, Yueyang Li: None declared, Timothy Varghese: None declared Janet Pope Grant/research support from: AbbVie, Bristol-Myers Squibb, Eli Lilly \& Company, Merck, Roche, Seattle Genetics, UCB, Consultant of: AbbVie, Actelion, Amgen, Bayer, Boehringer Ingelheim, Bristol-Myers Squibb, Eicos Sciences, Eli Lilly \& Company, Emerald, Gilead Sciences, Inc., Janssen, Merck, Novartis, Pfizer, Roche, Sandoz, Sanofi, UCB, Speakers bureau: UCB

DOI: 10.1136/annrheumdis-2020-eular.5136

\section{SAT0458 1 THE POLYMORPHISM ON FAT MASS AND OBESITY ASSOCIATED GENE (FTO) WAS ASSOCIATED WITH LOW BONE MASS IN JAPANESE COMMUNITY DWELLING POPULATION.}

K. Arima ${ }^{1}$, M. Tamai ${ }^{2}$, F. Nonaka ${ }^{3}$, N. Imamoto ${ }^{2}$, S. Mizukami ${ }^{1}$, T. Nishimura ${ }^{1}$, Y. Abe ${ }^{1}$, T. Origuchi ${ }^{4}$, K. Aoyagi ${ }^{1} .{ }^{1}$ Nagasaki University Graduate School of Biomedical Sciences, Department of Public Health, Nagasaki, Japan; ${ }^{2}$ Nagasaki University Graduate School of Biomedical Sciences, Department of Immunology and Rheumatology, Division of Advanced Preventive Medical Sciences, Nagasaki, Japan; ${ }^{3}$ Nagasaki University Graduate School of Biomedical Sciences, Department of Island and Rural Medical Research, Nagasaki, Japan; ${ }^{4}$ Nagasaki University Graduate School of Biomedical Sciences, Department of Rehabilitation Sciences, Nagasaki, Japan

Background: A polymorphism on FTO (OMIM 610966) was reported as a causa variant for obesity, plays a critical rule not only in amount of adipose tissue but also in function of mitochondrial thermogenesis 1.

Objectives: To examine the association of the genotype on a single nucleotide polymorphism on FTO with bone health.

Methods: FTO rs1421085 polymorphism was genotyped in 1,601 community-dwelling Japanese participants. This cross-sectional study was nested in Nagasaki Islands Study, which is a prospective cohort in Goto City, in islands of Japan. Participants were recruited at medical check-ups for community dwelling population.

Bone mass of the calcaneus was evaluated with stiffness index calculated using a quantitative ultrasound measurement. Low bone mass was defined as a stiffness index less than $80 \%$ of the young adult mean.

Sera and peripheral blood mononuclear cells were obtained. The SNP of rs1421085 was genotyped using hydrolysis probe. The chi-squared test was used to determine whether the variants were in equilibrium in that population. Trend for the median of BMI among genotypes was assessed using the Jonckheere-Terpstra test. Potential associations between FTO polymorphism and overweight and between the polymorphism and low bone mass were evaluated using logistic regression. All analyses were carried out using SPSS 23.

Results: Genotype and allele frequencies for the polymorphism were in Hardy-Weinberg equilibrium (minor allele frequency $0.16, p=0.40$ ) in $1,601 \mathrm{com}$ munity-dwelling persons (mean age was 68.5 years in men and 68.1 years in women).

There were significant associations between the minor allele and higher median of BMI on dose dependent manner in men $(p=0.04$ for trend in men and $p=0.10$ 